\title{
Shaded-Mask Filtering for Extended Depth-of-Field Microscopy
}

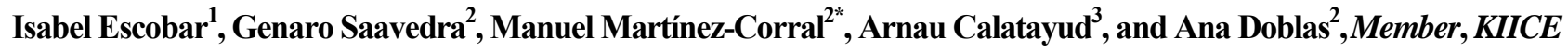 \\ ${ }^{1}$ Department of Applied Physics, University of Castilla la Mancha, Cuenca E-16071, Spain \\ ${ }^{2}$ Department of Optics, University of Valencia, Burjassot E-46100, Spain \\ ${ }^{3}$ Centro de Tecnología Físicas, Polytechnic University of Valencia, Valencia E-46022, Spain
}

\begin{abstract}
This paper proposes a new spatial filtering approach for increasing the depth-of-field (DOF) of imaging systems, which is very useful for obtaining sharp images for a wide range of axial positions of the object. Many different techniques have been reported to increase the depth of field. However the main advantage in our method is its simplicity, since we propose the use of purely absorbing beam-shaping elements, which allows a high focal depth with a minimum modification of the optical architecture. In the filter design, we have used the analogy between the axial behavior of a system with spherical aberration and the transverse impulse response of a 1D defocused system. This allowed us the design of a ring-shaded filter. Finally, experimental verification of the theoretical statements is also provided.
\end{abstract}

Index Terms: Aberrations, Apertures, Diffraction theory

\section{INTRODUCTION}

Imaging systems with a high depth-of-field (DOF) are required in many applications across different fields [1-5], such as microscopy [6-8] and communications [9]. However, most imaging systems described in the literature are very sensitive to defocusing. This means that small misalignments between the object and image plane impose great limitations on the imaging systems. In order to increase the DOF of these systems, numerous studies have been carried out recently along these lines [10-16].

The trivial method for increasing the DOF is to reduce the numerical aperture (NA) of imaging systems. However, this provokes a dramatic decrease in the transverse resolution: there exists a compromise between the transverse resolution and DOF. Thus, considerable effort has been expended in attempting to increase the DOF of imaging systems without undermining their resolution. In a particular case of the extension of the DOF while maintaining the transverse resolution [8, 13], many pupil masks have been designed based on phase [6, 7, 13-15, 17, 18] and/or amplitude [19-21]. It is of interest that the advantages of phase masks are superior to those of amplitude masks, including the fact that the most recently developed amplitude masks are not sufficiently light efficient. Another technique is the utilization of a multifocal concept, which uses various lenses of different focuses [22].

In this work, we analyze the enhancement of DOF in imaging systems. For this, we employ the analogy between the axial behavior of a system affected by spherical aberration and the transverse response of an imaging system. To study the increase in the DOF, we implement an

Received 10 March 2013, Revised 02 April 2013, Accepted 15 April 2013

*Corresponding Author Manuel Martínez-Corral (E-mail: manuel.martinez@uv.es, Tel: +34 96354 4718)

Department of Optics, University of Valencia, Av. Blasco Ibáñez 13, Burjassot E-46100, Spain.

(c) This is an Open Access article distributed under the terms of the Creative Commons Attribution Non-Commercial License (http://creativecommons.org/li-censes/by$\mathrm{nc} / 3.0 /$ ) which permits unrestricted non-commercial use, distribution, and reproduction in any medium, provided the original work is properly cited. 
amplitude filter in two optical systems. Similarly, any other filter designed to reduce the spherical aberration can be used to increase the DOF.

The paper is organized as follows. In Section II, we derive the equations that describe an optical system affected by spherical aberration. Also, as a specific case worth analyzing, the complex amplitude distribution is particularized to study the axial behavior of the imaging system. Section III is devoted to demonstrating the similarity between the axial response of an imaging system with spherical aberration and the transverse response of an optical system. Finally, in Section IV, we set up two different experiments. The first of them is implemented in an imaging system whose NA is low. For this case, we show both the numerical and experimental verification; the high agreement between them is clear. The second experiment is performed with conventional scanning microscopy. To sum up, in Section V, we conclude the main achievements of our research.

\section{THE MISMATCH INDEX-INDUCED SPHERICAL ABERRATION}

In this section, we study the aberration in a high-NA system when the wave field is focused through several media stacked perpendicularly to the optical axis.

Let us start by considering a high-NA objective lens illuminated by a monochromatic collimated beam with wavelength $\lambda$. The geometry of the objective is illustrated in Fig. 1. Contrary to what happens in the paraxial approach, the objective is characterized by its principal surfaces, which are a planar surface, $S_{1}$, and a spherical surface $S_{2}$, with focus $f$ and centered at the focal point, F. In most of the high-NA objectives reported in the literature, the aperture stop is located at the back-focal plane. Thus, if a monochromatic planar wave strikes the objective lens, the emerging wave field is a truncated spherical wavefront [23]. This wavefront is focused passing through a dielectric layer, the coverslip, whose thickness is $t$ and refractive index $n$, immersed into a medium with a different refractive index.

The amplitude distribution at the neighborhood of the focal plane can be calculated according the scalar, nonparaxial Debye's formulation [24] and assuming that the sine condition [25, 26] holds. After straightforward maths, the complex amplitude distribution along the optical axis is given by [27]

$$
U\left(w_{40}, w_{20}\right)=\int_{-0.5}^{0.5} q(\zeta) \exp \left(i 2 \pi w_{40} \zeta\right) \exp \left(-i 2 \pi w_{20} \zeta\right) \mathrm{d} \zeta,(1)
$$

where $w_{40}$ and $w_{20}$ are, respectively, the well-known spherical-aberration coefficient and defocus coefficient, as measured in units of wavelength, and $q(\zeta)$ is the apodized amplitude transmittance of the aperture stop.

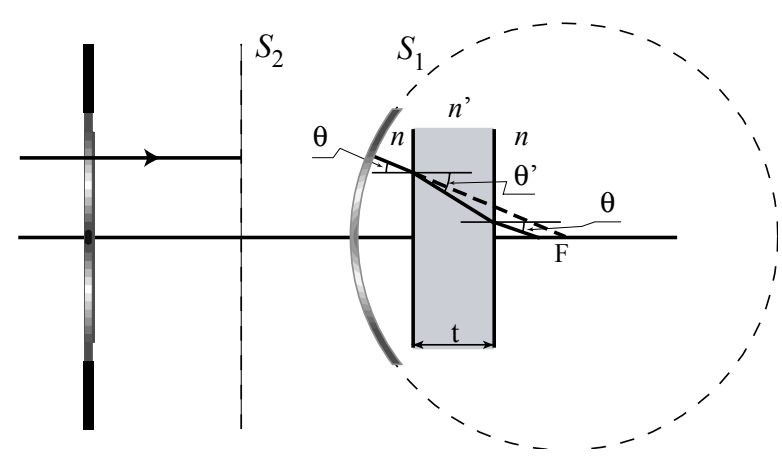

Fig. 1. Conceptual diagram to explain the focus process in a high numerical aperture objective into two media separated by a planar interface.

\section{DESIGN OF BEAM-SHAPING ELEMENTS TO INCREASE THE DEPTH-OF-FIELD}

Our aim here is to study beam-shaping elements that increase the DOF of optical systems. For that, let us consider a conventional two-dimensional (2D) imaging system, which basically consists of a telecentric arrangement, as shown in Fig. 2.

The telecentricity provides two important properties to the system: the system is $2 \mathrm{D}$ linear and shift-invariant. Therefore, the $2 \mathrm{D}$ irradiance distribution at the image space can be expressed as the $2 \mathrm{D}$ convolution between a scaled version of the $2 \mathrm{D}$ object and a $2 \mathrm{D}$ function, which is called the intensity point-spread function (PSF) of the imaging system [28],

$$
I(x, y ; z)=\frac{1}{M^{2}} O\left(\frac{x}{M}, \frac{y}{M}\right) \otimes_{2}\left|h^{\prime}(x, y ; z)\right|^{2}
$$

where $M=-f_{2} / f_{1}$ is the magnification of the imaging system and the intensity PSF is then obtained as the square modulus of the amplitude PSF

$$
\begin{aligned}
h^{\prime}(x, y ; z) & =\iint p\left(x_{p}, y_{p}\right) \exp \left[-i \frac{k z}{2 f^{2}}\left(x_{p}^{2}+y_{p}^{2}\right)\right] \times \\
& \exp \left[-i \frac{2 \pi}{\lambda f}\left(x x_{p}+y y_{p}\right)\right] \mathrm{d} x_{p} \mathrm{~d} y_{p},
\end{aligned}
$$

where, $p\left(x_{p}, y_{p}\right)$ represents the amplitude transmittance of the aperture stop (Fig. 2). We have also omitted some irrelevant factors. In the particular case in which the pupil function is separable in Cartesian coordinates, the amplitude PSF can be rewritten as $h^{\prime}(x, y ; z)=h_{x}^{\prime}(x ; z) h_{y}^{\prime}(y ; z)$, being 


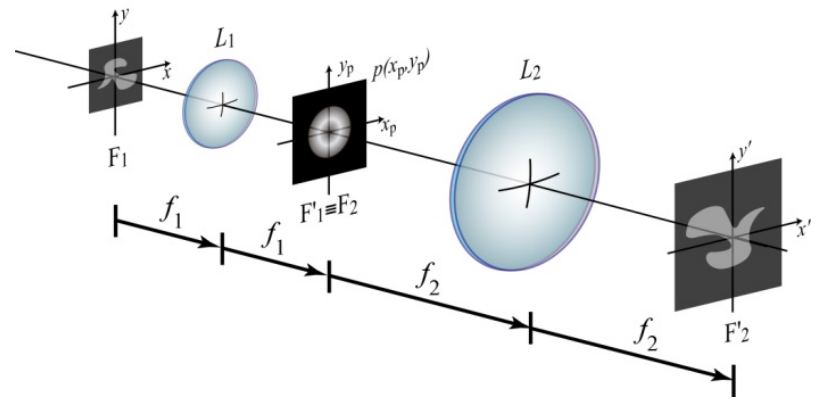

Fig. 2. Schematic of a two-dimensional telecentric imaging system. The light emanating from the object is collected by the objective $\left(L_{1}\right)$ and focused by the tube lens $\left(L_{2}\right)$.

$$
\begin{aligned}
h_{x}^{\prime}(x ; z)= & \int_{-0.5}^{0.5} p(\mu) \exp \left(-i \frac{2 \pi}{\lambda f^{2}} z r_{\max }^{2} \mu^{2}\right) \times \\
& \exp \left(-i \frac{2 \pi}{\lambda f} \sqrt{2} r_{\max } \mu\right) \mathrm{d} \mu .
\end{aligned}
$$

Note that the coordinates in the aperture stop plane have been normalized as

$$
\mu=\frac{x_{p}}{\sqrt{2} r_{\max }}
$$
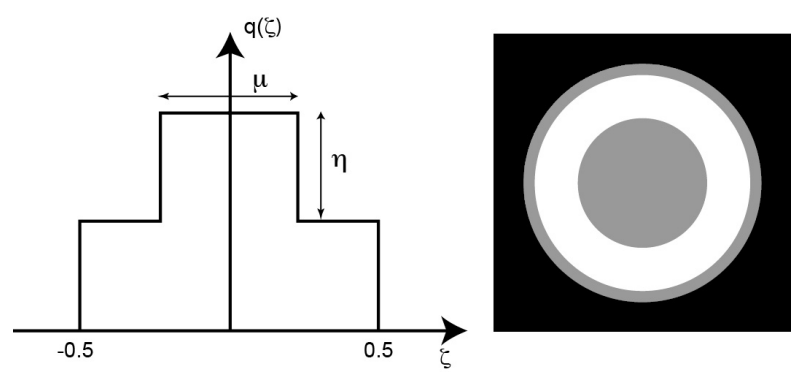

(a)

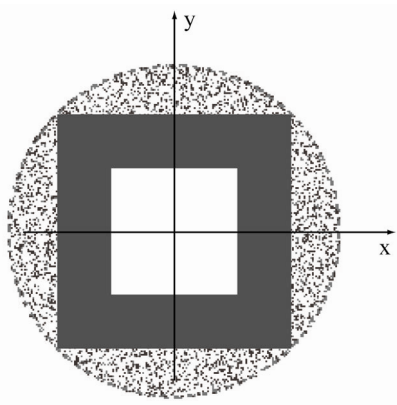

(b)

Fig. 3. (a) Shaded ring filter for reduction of the spherical aberration impact, (b) Cartesian version of the filter. where $r_{\max }$ is the radius of the circle in which the square pupil is inscribed.

It is interesting to note the similarity between Eqs. (1) and (4). This implies that the axial response of an imaging system affected by spherical aberration behaves similarly to the transverse response of an imaging system with a square pupil. This reasoning leads us to conclude that the amplitude profile family designed to reduce the spherical aberration may also be used to provide greater tolerance to defocusing in imaging systems.

The general case study can be particularized to the case of binary masks known as shaded ring (SR) filters. These filters are composed of three annular zones with two different transmittances, and each mask is uniquely specified by two construction parameters $(\mu, \eta)$ as defined in Fig. 3. From Fig. 3 , it is trivial to realize that a square filter produces a significant loss of resolution in certain transverse directions because the entire pupil size is not used. Consequently, a corresponding radial version has been designed (Fig. 4), where the transverse resolution is now the same in all directions. After a numerical optimization procedure [29], we have selected the values $\mu=0.4$ and $\eta=0.7$.

Obviously, to evaluate the PSF in this case, it is more convenient to rewrite Eq. (12) in cylindrical coordinates. Moreover, by employing the analogy with the axial response of an imaging system with spherical aberration, the amplitude PSF is

$$
h^{\prime}(r ; z)=\int_{0}^{1} p(\rho) \exp \left(i 2 \pi w_{20} \rho^{2}\right) J_{0}\left(\frac{2 \pi r_{\max }}{\lambda f} \rho r\right) \rho \mathrm{d} \rho,
$$

where $\rho=r_{p} / r_{\max }$ and the defocus coefficient is defined as

$$
w_{20}=-\frac{r_{\max }^{2} z}{2 \lambda f^{2}}
$$

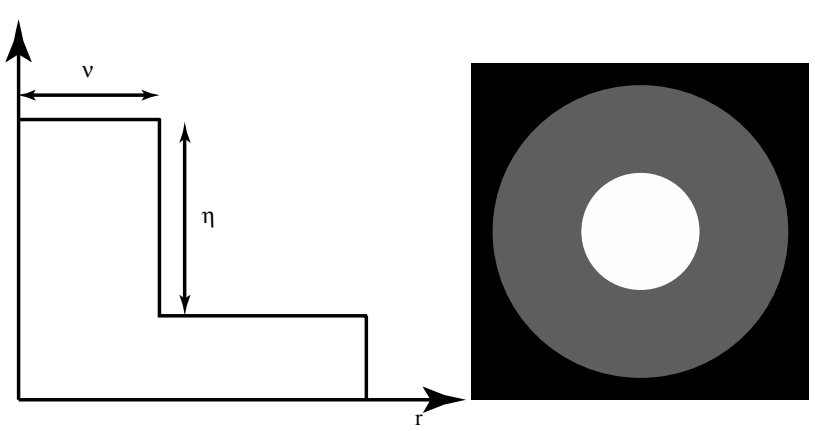

Fig. 4. Structure of the optimized shaded ring filter, called the defocus tolerance filter, which increases the depth-of-field. The two construction parameters are $\mu=0.4$ and $\eta=0.7$. 


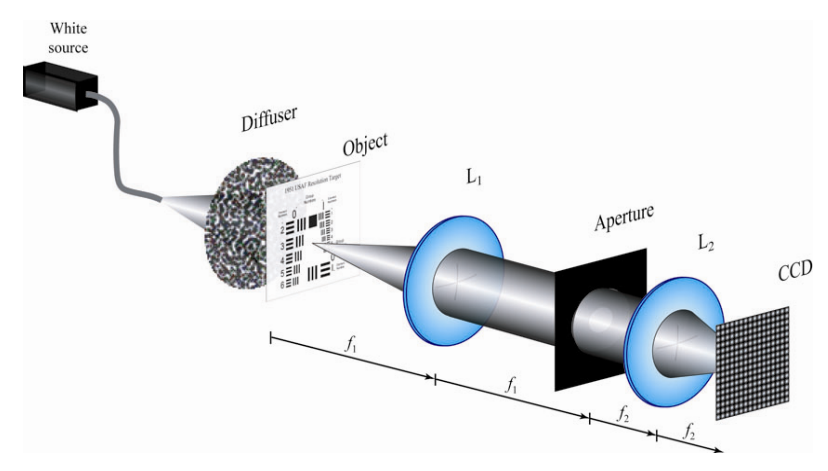

Fig. 5. Conceptual experiment to demonstrate the extended field of view in a low numerical aperture imaging system. The defocus tolerance filter is placed at the front focal plane of $L_{1}$.

\section{EXPERIMENTAL VERIFICATION}

To demonstrate the effects of defocusing in a low-NA imaging system apodized with both the clear aperture and an optimum SR filter, which is referred to as defocus tolerance (DT) filter, we prepared the experimental setup shown in Fig. 5. The DT filter was fabricated with highcontrast photographic film (Kodak Technical Pan; Rochester, NY, USA). For the illumination of a USAF 1951 resolution chart, we employed the diffused light proceeding from a white source.

In the setup of Fig. 5, the imaging system was operated in telecentric mode and was composed of two converging lenses whose focal lengths were $f_{1}=400 \mathrm{~mm}$ and $f_{2}=100$ $\mathrm{mm}$. According to this, the resolution test was placed at the front focal plane of $L_{l}$. To capture the images, we used a CCD camera (JAI/Pulnix TM-765E; Copenhagen, Denmark) composed of $765 \times 578$ square pixels of $11 \mu \mathrm{m}$ on each side.

In our experiment we recorded a set of $2 \mathrm{D}$ images at different axial positions, $z$. For simplicity, it is convenient to mount the CCD on a micrometric translation stage to provide a high-precision at different axial positions. Specifically, we selected $z=0,2.75,5.50$, and $8.25 \mathrm{~mm}$, which, according to Eq. (16), correspond to defocus coefficients of $w_{20}=0,-1,-2$, and -3 . Note that, theoretically, the PSF is symmetrical about the focal plane, so that we do not consider positive defocus parameters because we assume that we would obtain similar results.

In Figs. 6 and 7 we show the numerical and experimental results of the resolution target with the circular aperture and the DT filter. Clearly, the similarity between the experimental and calculated results is apparent. These figures also indicate that the greater the defocus coefficient, the smaller response of the clear aperture. Note that we cannot discern the low frequencies in the elements 1 and 2 of group 0 (1 and $2 \mathrm{LP} / \mathrm{mm}$, respectively) for $w_{20}=-3$. However, the response of the DT filter remains fairly stable and for $w_{20}$ $=-3$ it is possible to detect frequencies of $2.52 \mathrm{LP} / \mathrm{mm}$ corresponding to element 3 of group 1. Another fact to consider, in the case of a non-apodized system (left row of Fig. 7), is the contrast inversion in several elements of the test group 1 for $w_{20}=-2$.

Finally, to demonstrate the experimental case of high-NA, we arranged the experimental setup schematized in Fig. 8; this arrangement corresponds to a conventional scanning microscope. For this experiment, the light emerging from a fiber coupled to a He-Ne laser $(\lambda=632.8 \mathrm{~nm})$ was collimated through a converging lens of focal length $f_{L 1}=$ $200 \mathrm{~mm}$. After passing through a relay system and a beamsplitter, the wave field was focused via a microscope objective, whose NA was 0.9 , onto the sample.
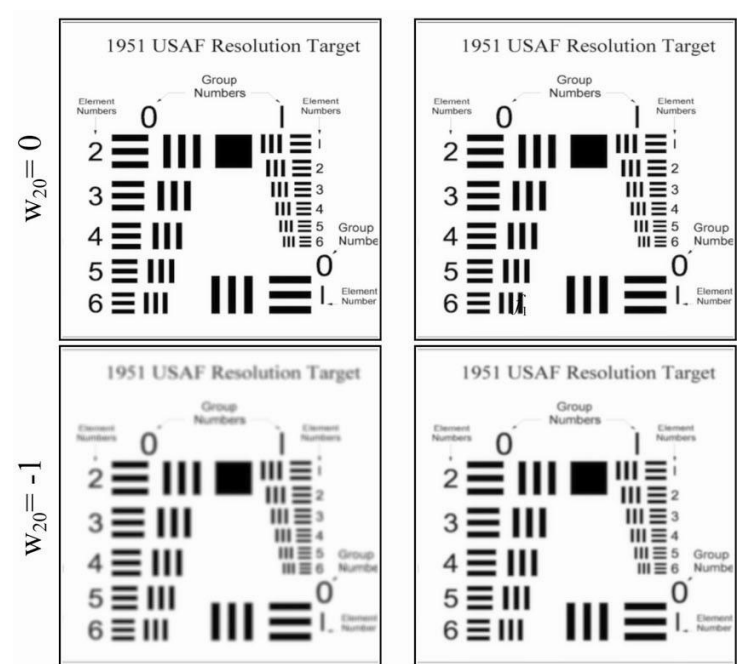

1951 USAF Resolution Target

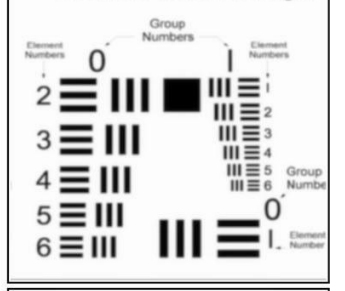

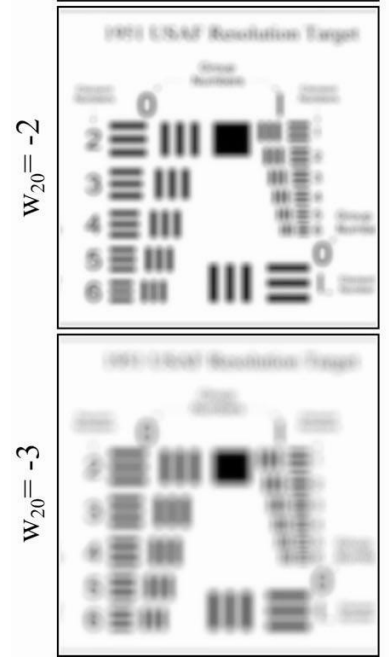

(a) Clear aperture

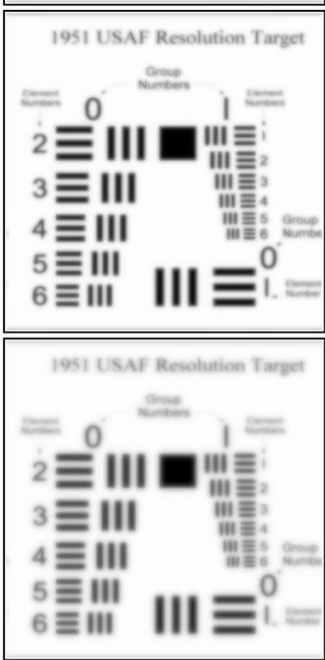

(b) DT filter

Fig. 6. Numerically-evaluated results corresponding to (a) the clear aperture and (b) the defocus tolerance (DT) filter for different values of the defocus coefficient $w_{20}$. As shown in the right column, the improvement with the depth-of-field is apparent. 
The signal reflected by the sample was finally focused onto a pinhole of radius of $50 \mu \mathrm{m}$.

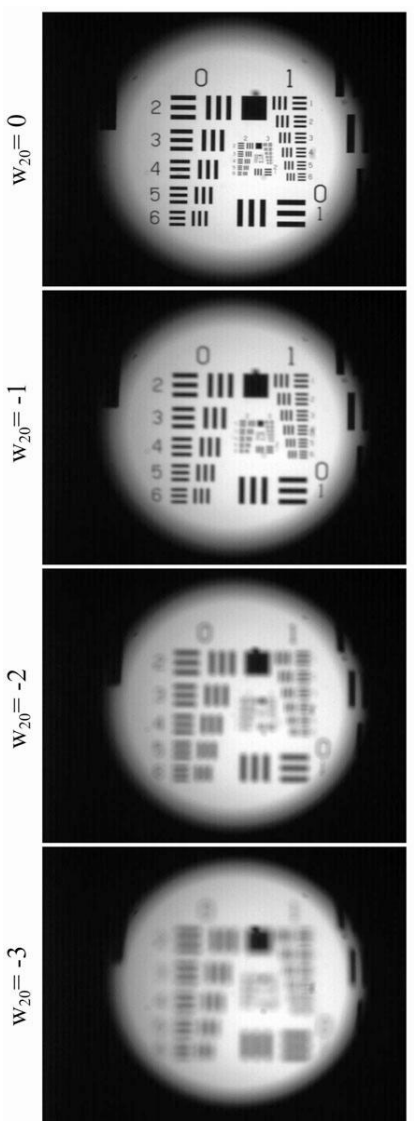

(a) Clear aperture
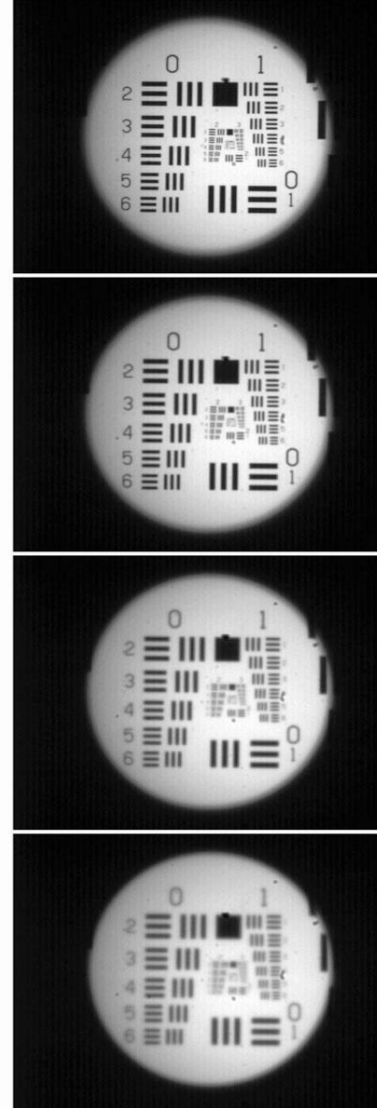

(b) DT filter
Fig. 7. Experimental results of a resolution chart corresponding to (a) the clear aperture and (b) the defocus tolerance (DT) filter. It is clear that the response of the DT filter remains fairly stable, and it is possible to detect higher frequencies with it.

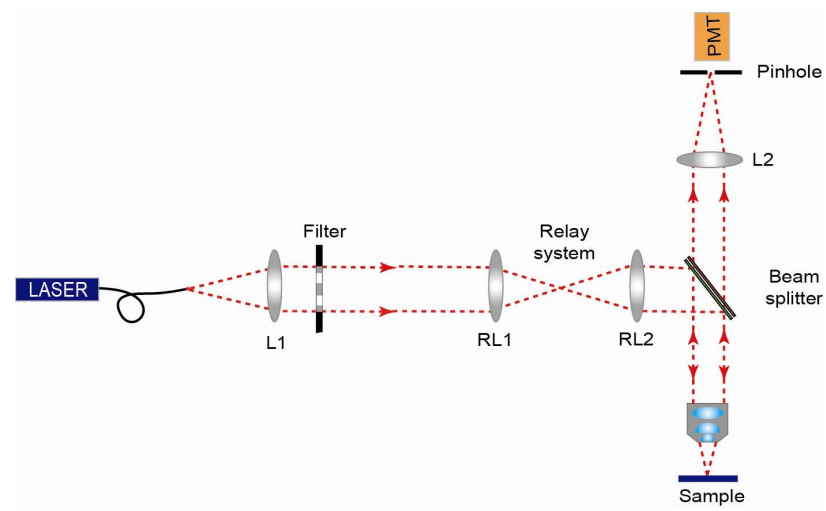

Fig. 8. Schematic layout of practical implementation of a conventional scanning microscope. The special feature of such an arrangement is the insertion of a relay system, which makes possible the introduction of an apodized filter.

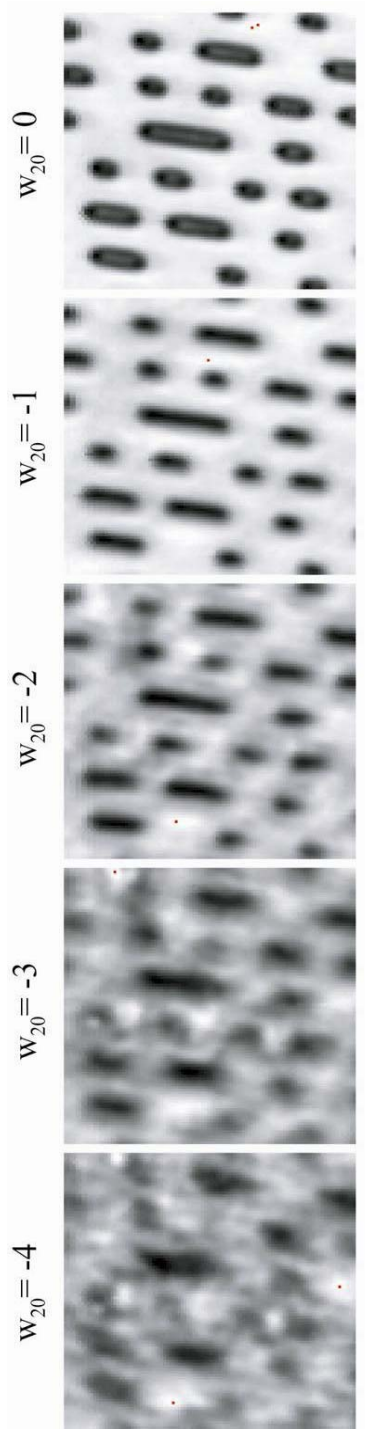

(a) Clear aperture

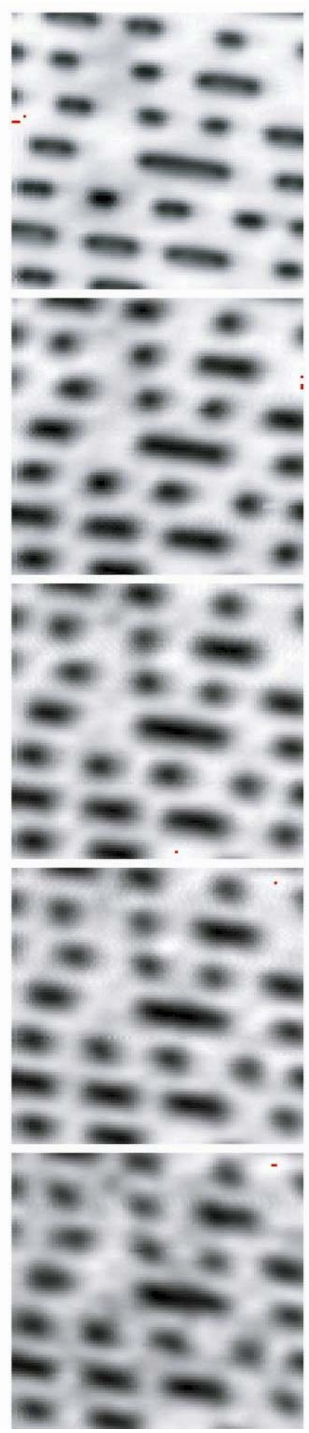

(b) DT filter
Fig. 9. Experimental verification of an extended depth-of-field for a high numerical aperture scanning microscope. For the measurement, we used the tracks on a $C D$ as the object. (a) Clear aperture, (b) the defocus tolerance (DT) filter.

We have carefully chosen the radius of the pinhole given that the detection was not confocal [30-33]. The pinhole was placed in front of a detector; in our case, it was a photomultiplier tube. A small fragment of an original music CD was imaged. This object was composed of a collection of tracks recorded on the CD.

Our goal was to increase the DOF. This task can be accomplished by modifying the exit pupil with the use of a DT filter. For this purpose, we used a relay system set up from RL1 $\left(f_{R L 1}=200 \mathrm{~mm}\right)$ and RL2 $\left(f_{R L 2}=175 \mathrm{~mm}\right)$.

In Fig. 9, we show the experimental results. In this case, an increase in one unit in $w_{20}$ value corresponds to an axial 
displacement of $z=1.12 \mu \mathrm{m}$. Again we can see that the DT filter provides very stable behavior as the defocus parameter increases. The DT filter significantly improves the image quality from values superior to -2 .

\section{CONCLUSIONS}

In summary, in this work it has been shown that the DOF has been increased in an imaging system affected by spherical aberration. This result opens the way to reducing the defocus in an imaging system by using an amplitude filter designed firstly to reduce spherical aberration. The improvement of DOF has been checked with an optimized SR filter (called a DT filter), which has been implemented in two types of experimental architecture. It should be noted that in both experiments, the DT filter provides very stable behavior compared to the clear aperture.

\section{ACKNOWLEDGMENTS}

This work was funded in part by the Ministerio de Economia y Competitividad, Spain, under Grant DPI201232994, and also by Generalitat Valenciana under Grant PROMETEO2009-077. Furthermore, A. Doblas acknowledges funding from the University of Valencia through the predoctoral fellowship program Atraccion de Talent.

\section{REFERENCES}

[ 1 ] S. Zhou and C. Zhou, "Discrete continuous-phase superresolving filters," Optics Letters, vol. 29, no. 23, pp. 2746-2746, 2004.

[2] M. Yun, L. Liu, J. Sun, and D. Liu, "Three-dimensional superresolution by three-zone complex pupil filters," Journal of the Optical Society of America A, vol. 22, no. 2, pp. 272-277, 2005.

[ 3 ] P. N. Gundu, E. Hack, and P. Rastogi, "Apodized superresolution concepts and simulations," Optics Communications, vol. 249, no. 1-3, pp. 101-107, 2005.

[ 4 ] S. F. Pereira and A. S. van de Nes, "Superresolution by means of polarization, phase and amplitude pupil masks," Optics Communications, vol. 234, no. 1-6, pp. 119-124, 2004.

[ 5 ] V. F. Canales, D. M. de Juana, and M. P. Cagigal, "Superresolution in compensated telescopes," Optics Letters, vol. 29, no. 9, pp. 935937, 2004.

[6] M. T. Caballero, P. Andres, A. Pons, J. Lancis, and M. MartinezCorral, "Axial resolution in two-color excitation fluorescence microscopy by phase-only binary apodization," Optics Communications, vol. 246, no. 4-6, pp. 313-321, 2005.

[ 7 ] D. M. de Juana, J. E. Oti, V. F. Canales, and M. P. Cagigal, "Transverse or axial superresolution in a 4Pi-confocal microscope by phase-only filters," Journal of the Optical Society of America A, vol. 20, no. 11, pp. 2172-2178, 2003

[ 8 ] L. Liu, C. Liu, W. C. Howe, C. J. R. Sheppard, and N. Chen, "Binary-phase spatial filter for real-time swept-source optical coherence microscopy," Optics Letters, vol. 32, no. 16, pp. 2375$2377,2007$.

[9] J. Jia, C. Zhou, and L. Liu, "Superresolution technology for reduction of the far-field diffraction spot size in the laser freespace communication system," Optics Communications, vol. 228, no. 4-6, pp. 271-278, 2003.

[10] J. Ojeda-Castaneda, S. Ledesma, and C. M. Gomez-Sarabia, "Hyper Gaussian windows with fractional wavefronts," Photonics Letters of Poland, vol. 5, no. 1, pp. 23-25, 2013.

[11] F. Diaz, F. Goudail, B. Loiseaux, and J. P. Huignard, "Design of a complex filter for depth of focus extension," Optics Letters, vol. 34, no. 8, pp. 1171-1173, 2009.

[12] M. A. Golub, V. Shurman, and I. Grossinger, "Extended focus diffractive optical element for Gaussian laser beams," Applied Optics, vol. 45, no. 1, pp.144-150, 2006.

[13] L. Liu, F. Diaz, L. Wang, B. Loiseaux, J. P. Huignard, C. J. R. Sheppard, and N. Chen, "Superresolution along extended depth of focus with binary-phase filter for the Gaussian beam," Journal of the Optical Society of America A, vol. 25, no. 8, pp. 2095-2101, 2008.

[14] C. J. R. Sheppard, "Binary phase filter with a maximally-flat response," Optics Letters, vol. 36, no. 8, pp.1386-1388, 2011.

[15] C. J. R. Sheppard and S. Mehta, "Three-level for increased depth of focus and Bessel beam generation," Optics Express, vol. 20, no. 25, pp. 27212-27221, 2012.

[16] C. J. R. Sheppard, "Pupil filter for generation of light sheets," Optics Express, vol. 21, no.5, pp. 6339-6345, 2013.

[17] A. Castro, J. Ojeda-Castaneda, and A.W. Lohmann, "Bow-tie effect: differential operator," Applied Optics, vol. 45, no. 30, pp. 7878-7884, 2006

[18] Z. Zalevsky, A. Shemer, A. Zlotnik, E. B. Eliezer, and E. Marom, "All-optical axial super resolving imaging using a low-frequency binary-phase mask," Optics Express, vol. 14, no. 7, pp. 2631-2643, 2006.

[19] J. Ojeda-Castaneda, E. Tepichin, and A. Diaz, "Arbitrarily high focal depth with a quasioptimum real and positive transmittance apodizer," Applied Optics, vol. 28, no. 13, pp. 2666-2670, 1989.

[20] C. M. Hammond Jr, "Apparatus and method for reducing imaging errors in imaging systems having an extended depth of field," US patent 6097856, 2000.

[21] D. Miller and E. Blanco, "System and method for increasing the depth of focus of the human eye," US patent 6554424, 2003.

[22] E. Ben-Eliezer, E. Marom, N. Konforti, and Z. Zalevsky, "Experimental realization of an imaging system with an extended depth of field," Applied Optics, vol. 44, no. 14, pp. 2792-2798, 2005.

[23] C. J. R. Sheppard and M. Gu, "Imaging by a high aperture optical system," Journal of Modern Optics, vol. 40, no. 8, pp. 1631-1651, 1993.

[24] P. Debye, "Der lichtdruck auf kugeln von beliebigem material," 
Annalen der Physik, vol. 335, no. 11, pp. 57-136, 1909.

[25] M. Born and E. Wolf, Principles of Optics, 7th ed. New York, NY: Cambridge University Press, 1999.

[26] C. J. R. Sheppard and H. J. Matthews, "Imaging in high-aperture optical system," Journal of the Optical Society of America A, vol. 4, no. 8, pp. 1354-1360, 1987.

[27] I. Escobar, G. Saavedra, M. Martinez-Corral, and J. Lancis, "Reduction of the spherical aberration effect in high-numericalaperture optical scanning instruments," Journal of the Optical Society of America A, vol. 23, no. 12, pp. 3150-3155, 2006.

[28] M. Martinez-Corral and G. Saavedra, "The resolution challenge in 3D optical microscopy," Progress in Optics, vol. 53, pp. 1-68, 2009.

[29] I. Escobar, E. Sanchez-Ortiga, G. Saavedra, and M. MartinezCorral, "New analytical tools for evaluation of spherical aberration in optical microscopy," in Optical Fluorescence Microscopy: from the Spectral to the Nano Dimension, Heidelberg, Germany: Springer, pp. 85-99, 2011.

[30] T. Wilson and A. R. Carlini, "Three-dimensional imaging in confocal imaging-systems with finite sized detectors," Journal of Microscopy, vol. 149, no. 1, pp. 51-66, 1988.

[31] S. Kimura and C. Munakata, "Dependence of 3-D optical transfer functions in the pinhole radius in a fluorescent confocal optical microscope," Applied Optics, vol. 29, no. 20, pp. 3007-3011, 1990.

[32] M. Gu and C. J. R. Sheppard, "Effects of a finite-sized pinhole on 3D image formation in confocal two-photon fluorescence microscopy," Journal of Modern Optics, vol. 40, no. 10, pp. 20092024, 1993.

[33] R. Gauderon and C. J. R. Sheppard, "Effect of a finite-size pinhole on noise performance in single-, two-, and three-photon confocal fluorescence microscopy," Applied Optics, vol. 38, no. 16, pp. 3562-3565, 1999.

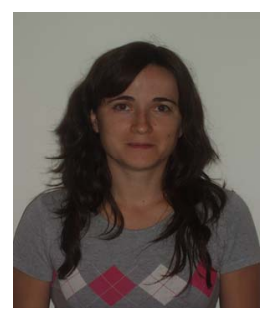

\section{Isabel Escobar}

received the M.Sc. and Ph.D. degrees in physics from the University of Valencia in 2003 and 2008, respectively. She is currently Assistant Professor of Physics at the University of Castilla la Mancha. Her teaching includes lectures and supervision of laboratory experiments for undergraduate students in telecommunications engineering and architectural engineering. Her research topics are mainly related to 3D microscopy, along with acoustic impedance and absorption coefficients of building materials.

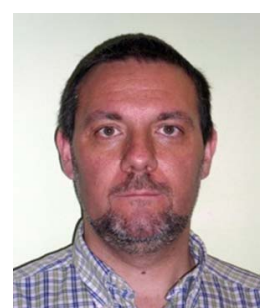

\section{Genaro Saavedra}

was born in Spain in 1967. He received his B.Sc. and Ph.D. degrees in physics from the University of Valencia, Spain, in 1990 and 1996, respectively. He is currently Full Professor with this university, and co-leads the 3D Imaging and Display Laboratory. His current research interests are optical diffraction, integral imaging, 3D high-resolution optical microscopy, and phase-space representation of scalar optical fields. He has published on these topics about 50 technical articles in major journals and three chapters in scientific books. He has published over 50 conference proceedings, including 10 invited presentations.

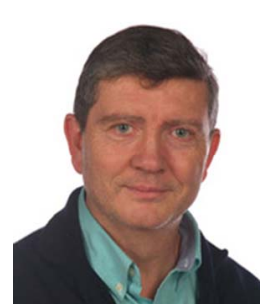

\section{Manuel Martinez-Corral}

was born in Spain in 1962. He received the M.Sc. and Ph.D. degrees in physics from the University of Valencia, Spain, in 1988 and 1993, respectively. He is currently Full Professor of Optics at the University of Valencia, where he co-leads the 3D Imaging and Display Laboratory. Since 2010, he has been a Fellow of SPIE. His research interests include scalar and vector properties of tightly focused light fields, resolution procedures in 3D scanning microscopy, and $3 \mathrm{D}$ imaging and display technologies. He has published over 75 technical articles in major journals, and has given over 30 invited and keynote presentations in international meetings. He has been a member of the Scientific Committee for more than 20 international meetings. He is Topical Editor of the IEEE/OSA Journal of Display Technology and Associate Editor of the Journal of Information and Communication Convergence Engineering.

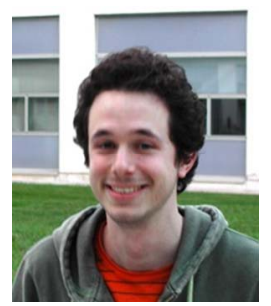

\section{Arnau Calatayud}

was born in Valencia in 1984. He received the B.Sc. degree in Physics and M.Sc. degree in Optics from the Universidad de Valencia, Spain. He received an Electrical Engineering degree from the Universidad de Valencia in 2011. Since May 2009 he has been working towards a Ph.D. degree in Physics and holds a pre-doctoral fellowship at the Polytechnic University of Valencia. His current main interest is optical lens design. 


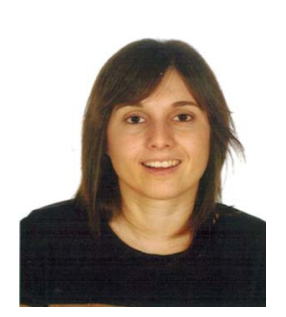

Ana Doblas

was born in Spain in 1987. She received the B.Sc. and M.Sc. degrees in Physics from the University of Valencia, Spain, in 2010 and 2011, respectively. Since 2009, she has been working with the 3D Imaging and Display Laboratory (University of Valencia), where she is currently running her PhD project. Her research interests include 3D optical microscopy and image formation theory. 